\title{
Energy balances for propagating gravity currents: homogeneous and stratified ambients
}

\author{
By MARIUS UNGARISH ${ }^{1}$ AND HERBERT E. HUPPERT ${ }^{2}$ \\ ${ }^{1}$ Department of Computer Science, Technion, Haifa 32000, Israel \\ ${ }^{2}$ Institute of Theoretical Geophysics, DAMTP University of Cambridge, Wilberforce Rd, \\ Cambridge CB3 0WA, UK
}

(Received 8 August 2005 and in revised form 28 March 2006)

The exchange of energy for an inviscid gravity current which is released from a lock and then propagates over a horizontal boundary is considered. Attention is focused on effects due to stratification in the ambient. The investigation uses both a one-layer shallow-water model and Navier-Stokes finite-difference simulations. There is good agreement between these two approaches for the energy of the dense fluid (the current). The results indicate that with respect to the behaviour of energy as a function of time we can distinguish between: (a) currents propagating at supercritical speed (with respect to the fastest internal wave in the ambient), including a nose propagating into an unstratified ambient; and $(b)$ currents propagating at subcritical speed, including the strongest effective stratification for which the density at the base of the ambient is equal to that of the current. The stratification enhances the accumulation of potential energy in the ambient and reduces the energy decay (dissipation) of the two-fluid system. The interaction of the internal waves with the head of the current in the subcritical case has no significant influence on the energy balance of the current.

\section{Introduction}

Gravity currents occur whenever fluid of one density flows primarily horizontally into fluid of a different density. Many such situations arise in both industrial and natural settings, as reviewed by Simpson (1997) and Huppert (2000). Various important features of these processes have now been fairly well investigated. Our aim here is primarily to elucidate the energy flow during the propagation of highReynolds-number currents, such as those resulting from the instantaneous release of a finite volume of constant density in a rectangular two-dimensional geometry. We are particularly interested in effects due to a stratified ambient. Applications of our work include areas such as oceanography, atmospheric winds and environmental control. For example, submarine wakes in oceans, contrails in the atmosphere, and the crests of lee waves in air streams over a mountain are typically regions of 'mixed' fluid whose density difference from the ambient produces a driving force of the type considered here, see $\mathrm{Wu}(1969)$.

The study of gravity currents and intrusions into a stratified ambient has made significant progress in the last few years. Earlier works aimed at the elucidation of the high-Reynolds-number motion of a fixed volume of fluid released from a lock were concerned mostly with intrusions at the level of neutral buoyancy (Wu 1969; Kao 1976; Manins 1976; Amen \& Maxworthy 1980; Faust \& Plate 1984; de Rooij 1999). 
(Related investigations concerning the waves produced in the ambient were presented by Schooley \& Hughes 1972; Maxworthy 1980, 1983; Flynn \& Sutherland 2004, but this phenomenon is beyond the scope of the present work.) The pertinent theoretical interpretations, based on box models with adjustable parameters, turned out to have a quite restricted range of applicability. For example, Faust \& Plate (1984) on the basis of careful comparisons with their experimental data, concluded that 'intrusions into a linearly stratified environment behave very differently from theoretical calculations'. A turning point came with the work of Maxworthy et al. (2002), herein referred to as M. They considered the propagation of a saline current released from behind a lock over a horizontal bottom into a linearly stratified saline ambient in a rectangular container whose upper boundary was open to the atmosphere. The density of the current, $\rho_{c}$, was larger than, or equal to, that of the ambient at the bottom, $\rho_{b}$ (the 'intrusion' corresponds to the particular case $\rho_{c}=\rho_{b}$ ). The investigation was a combination of laboratory and numerical experiments. $\mathrm{M}$ observed that the speed of propagation is time independent for a significant period after release (following a short adjustment interval). They obtained a quite general empirical data correlation for this slumping velocity as a function of two governing dimensional parameters: $S$ (which expresses the strength of the stratification defined by (2.2)) and $H$ (which expresses the depth ratio of the ambient to the lock). $\mathrm{M}$ also provide data on the 'criticality' of the speed (with respect to the fastest internal wave in the ambient) and the position where the first significant wave-nose interaction is observed. Motivated by the lack of theoretical interpretation of the careful observations of M, Ungarish \& Huppert $(2002,2004)$ and Ungarish (2005a) developed and verified the corresponding one-layer inviscid shallowwater (SW) formulation. This theory is based on rigorous volume and momentum balances, which are reduced to a hyperbolic system of equations for the thickness $h$ and velocity $u$ of the current as functions of $x$ (horizontal distance) and $t$ (time), amenable to realistic initial and boundary conditions, without using any adjustable parameters. It provides reliable theoretical interpretations of the observations of $\mathrm{M}$. Indeed, the theory shows that: $(a)$ the governing parameters are $S$ and $H ;(b)$ the solution on the characteristics of the hyperbolic SW system yield initial propagation with constant velocity, at values in excellent agreement with measurements; and (c) the super- and subcritical speed of propagation as functions of the governing parameters $S$ and $H$, and the occurrence of the first wave-nose interaction can be predicted. Moreover, extensions of this SW formulation to axisymmetric and rotating currents were developed and the parameters which govern the stratification-Coriolis interactions were derived. Extensions to the flow of intrusions were presented by Ungarish (2005b). The new results are in very good agreement with the measurements of Faust \& Plate (1984), thus resolving the theory-experiment incompatibility dilemma pointed out by these authors. The new results also clarified the previously overlooked differences between intrusions released from behind a rectangular lock (Amen \& Maxworthy 1980; de Rooij 1999) and a cylinder lock (Wu 1969), and proved that the propagation of an intrusion is always subcritical in a linearly stratified ambient (the last result strengthens an earlier approximate deduction by Flynn \& Sutherland 2004). The propagation proportional to $t^{1 / 2}$, indicated by the experiments of $\mathrm{Wu}$, turns out to be a similarity solution of the SW balances. Ungarish (2006) generalized the nose Froude and dissipation analysis of Benjamin (1968) to a linearly stratified ambient, and showed that the classical unstratified results are fully recovered in the limit $S \rightarrow 0$. The useful insights gained by these studies and the good agreements with available experimental data provide motivation for deriving further results and verifications in the framework of the SW formulation. 

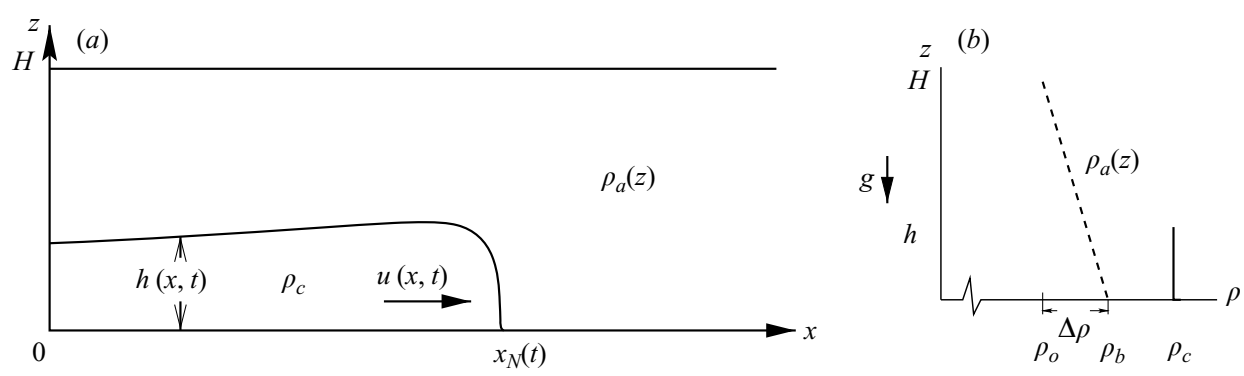

FiguRE 1. Schematic description of the system: $(a)$ the geometry; and $(b)$ the density profiles in the current (solid line) and ambient (dashed line).

The behaviour of the energy of the current has not been addressed directly in these investigations. This is an important issue for understanding the interaction between the current and the ambient and the behaviour of various natural hazards. In particular, it is important to clarify: the influence of stratification on the behaviour of the energy of the current; and whether the one-layer SW model, which neglects motion in the ambient, reproduces well the energy budgets of the current. The object of this paper is to study these questions. The investigation is based on a combination of SW and Navier-Stokes (NS) solutions for a two-dimensional (rectangular) geometry.

The system under consideration is sketched in figure 1: a deep layer of ambient fluid, of density $\rho_{a}(z)$, lies above a horizontal surface at $z=0$. Gravity acts in the negative $z$-direction. In the rectangular case the system is bounded by vertical, smooth, impermeable surfaces and the current propagates in the $x$-direction. At time $t=0$ a given volume of homogeneous fluid of density $\rho_{c} \geqslant \rho_{a}(0) \equiv \rho_{b}$ and kinematic viscosity $v$, initially at rest in a rectangular box of height $h_{0}$ and length $x_{0}$, is instantaneously released into the ambient fluid. A two-dimensional current starts to spread. We assume that the Reynolds number of the horizontal flow, $R e$, defined below, is large, and hence viscous effects can be neglected. (For example, in the experiments of $\mathrm{M}, R e$ was estimated as typically $10^{3}$. After a significant spread of the current, when both its thickness and the velocity are reduced, viscous forces become important. This phase is outside the scope of the present work.)

A related work, on the energy budgets of gravity currents which propagate into a homogeneous ambient, was recently presented by Necker et al. (2005). This is a careful numerical investigation, based on a high-resolution code. However, their focus was different from the present one: most attention was devoted to the effects of particle-driven currents, but the study did not consider SW models and stratified ambients.

The structure of the paper is as follows. In $\S 2$ the energy balances are developed, first in the SW model and then in the NS formulation. A criterion for the validity of the inviscid assumption is also introduced. In $\S 3$ we present results and comparisons of the SW and NS calculations for typical configurations. We show that, overall, the SW model captures well the features of the energy transfers. In $\S 4$ we present some concluding remarks about the effects of stratification. In particular, we note the difference between supercritical currents (including currents propagating into a non-stratified ambient) and subcritical currents. We also claim that the stratification of the ambient enhances the potential (wave) energy accumulation, and hence reduces the viscous dissipation (which acts on a reduced amount of kinetic energy). 


\section{Formulations}

The configuration is sketched in figure 1 . We use an $\{x, y, z\}$ Cartesian coordinate system with corresponding $\{u, v, w\}$ velocity components, assuming that the flow does not depend on the coordinate $y$ and that $v \equiv 0$.

Initially, the height of the propagating current is $h_{0}$, its length is $x_{0}$ and its density $\rho_{c}$. The height of the ambient fluid is $H$ (dimensional). The ambient fluid is stably stratified: the density at the top (usually, an open boundary) is $\rho_{o}$, and it increases linearly with depth by the increment $\Delta \rho$ to the value $\rho_{b}$ at the bottom. We consider situations with $\rho_{b} \leqslant \rho_{c}$.

It is convenient to use $\rho_{o}$ as the reference density and to introduce the reduced density differences and ratios between them (Ungarish \& Huppert 2002):

$$
\epsilon=\frac{\rho_{c}-\rho_{o}}{\rho_{o}}, \quad \epsilon_{b}=\frac{\rho_{b}-\rho_{o}}{\rho_{o}}=\frac{\Delta \rho}{\rho_{o}}
$$

and

$$
S=\epsilon_{b} / \epsilon,
$$

from which it follows that

$$
\rho_{c}=\rho_{o}(1+\epsilon), \quad \rho_{a}=\rho_{b}-\frac{\Delta \rho}{H} z=\rho_{o}\left[1+\epsilon S\left(1-\frac{z}{H}\right)\right] .
$$

$S$ represents the magnitude of the stratification in the ambient fluid, and we consider only $0 \leqslant S \leqslant 1$. The homogeneous ambient is recovered by setting $S=0$. We also define the reference reduced gravity,

$$
g^{\prime}=\epsilon g
$$

where $g$ is the gravitational acceleration.

We recall that the buoyancy frequency is defined by

$$
\mathscr{N}^{2}=g \frac{\Delta \rho}{\rho_{o} H}=g^{\prime} \frac{S}{H}
$$

and that the leading, or mode one, linear internal wave in a closed two-dimensional channel propagates with velocity (Baines 1995)

$$
u_{W}= \pm \frac{\mathscr{N} H}{\pi} .
$$

It is convenient for purposes of interpretation to keep in mind the following picture, also sketched in figure $1(b): S=0$ corresponds to a homogeneous ambient of density $\rho_{o}$ and a current of fluid of density $\rho_{c}$. For $0<S<1$ the density of the ambient is stratified (increases linearly) from the same $\rho_{o}$ at the top to a larger density at the bottom. The extreme situation $S=1$ is achieved when the density of the ambient at the base matches that of the current.

\subsection{SW model}

We shall use a one-layer approximation which omits the motion in the ambient. This is the simplest shallow-water model. In fact, the propagation of the current at the bottom is bound to produce a return flow in the ambient above. Experimental and numerical results (Wu 1969; M; Ungarish \& Huppert 2004; Ungarish 2005b) show that this flow has a quite complex $z$-dependence. Therefore, in contrast with the unstratified case, the flow field in the stratified ambient cannot be directly expressed by averaged variables. A reliable two-layer model for the stratified configuration 
is still lacking. Thus, although the one-layer model contains a bold simplification, at present it is the only available framework of governing equations for analytical investigation. Previous studies indicated that it captures well many of the important features of the flow, and we therefore claim that it makes sense to use it for energy calculations.

To be specific, we assume that in the ambient fluid domain $u=v=w=0$ and hence the fluid is in purely hydrostatic balance and maintains the initial density $\rho_{a}(z)$ given by (2.3). Motion is assumed to take place in the lower layer only, $0 \leqslant x \leqslant x_{N}(t)$ and $0 \leqslant z \leqslant h(x, t)$, see figure 1 . As in the classical inviscid shallow-water analysis of a gravity current in a homogeneous ambient, we argue that the predominant vertical momentum balance in the current is hydrostatic and that viscous effects in the horizontal momentum balance are negligibly small (the quantitative criteria will be specified later in §2.1.2). Hence the motion is governed by a balance between pressure and inertia forces in this horizontal direction. As in the situation with a homogeneous ambient, an order-of-magnitude analysis indicates that the perturbation of the upper free surface introduced by the flow can be neglected when $\epsilon \ll 1$, as assumed here.

A relationship between the pressure fields and the height $h(x, t)$ can be obtained. The hydrostatic balances are $\partial p_{i} / \partial z=-\rho_{i} g$, where $i=a$ or $c$, and in the motionless ambient the pressure does not depend on $x$. Use of (2.3) then yields

$$
p_{a}(z, t)=-\rho_{o}\left[1+\epsilon S\left(1-\frac{1}{2} \frac{z}{H}\right)\right] g z+C
$$

and

$$
p_{c}(x, z, t)=-\rho_{o}(1+\epsilon) g z+f(x, t),
$$

where $C$ is a constant. Pressure continuity between the ambient and the current on the interface $z=h(x, t)$ determines the function $f(x, t)$ of (2.8) and we obtain, after some algebra,

$$
p_{c}(x, z, t)=-\rho_{o}(1+\epsilon) g z+\rho_{o} g^{\prime}\left[h-S\left(h-\frac{1}{2} \frac{h^{2}}{H}\right)\right]+C,
$$

and consequently

$$
\frac{\partial p_{c}}{\partial x}=\rho_{o} g^{\prime} \frac{\partial h}{\partial x}\left[1-S\left(1-\frac{h}{H}\right)\right] .
$$

The fact that $\partial p_{c} / \partial x$ is not a function of $z$ makes the $\mathrm{SW}$ equations for the stratified case a straightforward extension of the homogeneous situation. Indeed, the motion in the layer of dense fluid can be expressed by two dependent variables: the thickness of the layer, $h(x, t)$, and the (z-averaged) longitudinal velocity, $u(x, t)$. In this approximation, the vertical velocity in the layer is negligibly small compared with $u$ (formally, the ratio is of the order of magnitude of $\left.\left(h_{0} / x_{0}\right)^{2}\right)$. The equations of motion will be presented in $\S 2.1 .2$.

\subsubsection{Energy}

We now consider the energy in the dense-fluid domain. In the SW framework the significant speed of motion is represented by the $z$-independent average horizontal velocity, and hence the kinetic energy of the current (denoted by subscript $c$ ) is simply

$$
K_{c}(t)=\frac{1}{2} \rho_{c} \int_{0}^{x_{N}(t)} u^{2}(x, t) h(x, t) \mathrm{d} x .
$$

The vertical displacement of the dense fluid particles is resisted by the hydrostatic pressure of the embedding ambient fluid. The resulting buoyancy acceleration is 
$\left[\rho_{c}-\rho_{a}(z)\right] g$ and the corresponding work needed to move a unit volume from the bottom to some $z$, in the present linear $\rho_{a}(z)$, is $g\left[\left(\rho_{c}-\rho_{b}\right) z+(1 / 2) \Delta \rho z^{2} / H\right]$. The potential energy of the current is therefore

$$
P_{c}(t)=g \int_{0}^{x_{N}(t)} \mathrm{d} x \int_{0}^{h(x, t)}\left[\left(\rho_{c}-\rho_{b}\right) z+\frac{1}{2} \frac{\Delta \rho}{H} z^{2}\right] \mathrm{d} z=g \int_{0}^{x_{N}(t)}\left[\frac{1}{2}\left(\rho_{c}-\rho_{b}\right) h^{2}+\frac{1}{6} \frac{\Delta \rho}{H} h^{3}\right] \mathrm{d} x .
$$

Initially, at $t=0$, we have $h=h_{0}, x_{N}=x_{0}$ and $u=0$. Consequently, $K_{c}(0)=0$ and, using (2.1)-(2.4), we obtain

$$
P_{c}(0)=\left(\frac{1}{2} \rho_{o} x_{0} h_{0}^{2} g^{\prime}\right)\left[1-S+\frac{1}{3} \frac{S}{H / h_{0}}\right] .
$$

This indicates that energy in the problem under investigation is conveniently scaled with $(1 / 2) \rho_{o} x_{0} h_{0}^{2} g^{\prime}$, as is done in (2.14) below. For a particular system it is convenient to refer the energy to $P_{c}(0)$, as is done in $\S \S 3$ and 4 .

\subsubsection{Governing $S W$ equations}

It is convenient to scale the dimensional variables (denoted here by asterisks) as follows:

$$
\left\{x^{*}, z^{*}, h^{*}, H^{*}, t^{*}, u^{*}, E^{*}\right\}=\left\{x_{0} x, h_{0} z, h_{0} h, h_{0} H, T t, U u, \frac{1}{2} \rho_{o} U^{2} h_{0} x_{0} E\right\},
$$

where

$$
U=\left(h_{0} g^{\prime}\right)^{1 / 2} \text { and } \quad T=x_{0} / U .
$$

Here $h_{0}$ and $x_{0}$ are the initial height and length of the current, $U$ is a typical inertial velocity of propagation of the nose of the current and $T$ is a typical time period for longitudinal propagation over a typical distance $x_{0}$. The variable $E$ denotes the energy (per unit width $y$ ). Note that the horizontal and vertical lengths are scaled differently, which removes the initial aspect ratio $h_{0} / x_{0}$ from the SW analysis in the homogeneous circumstances (Ungarish \& Huppert 1999), and this applies also to the stratified case considered here. A representative Reynolds number of the current is defined as $R e=U h_{0} / v$, where $v$ is the kinematic viscosity, assumed constant in the system.

The relevant volume and momentum balances have been developed and verified in previous investigations (Ungarish \& Huppert 2002, 2004; Ungarish 2005a). Here we briefly mention the equations used in the present work.

In conservation form the continuity and momentum equations can be written as

$$
\frac{\partial h}{\partial t}+\frac{\partial}{\partial x}(u h)=0
$$

and

$$
\frac{\partial}{\partial t}(u h)+\frac{\partial}{\partial x}\left[u^{2} h+\frac{1}{2}(1-S) h^{2}+\frac{1}{3} S \frac{h^{3}}{H}\right]=0 .
$$

Following the standard procedure, we calculate the speeds of propagation of the characteristics

$$
\lambda_{ \pm}=u \pm\left[h\left(1-S+S \frac{h}{H}\right)\right]^{1 / 2} .
$$


On $\mathrm{d} x / \mathrm{d} t=\lambda_{ \pm}$, the dependent variables satisfy

$$
\left[\frac{1-S+S(h / H)}{h}\right]^{1 / 2} \mathrm{~d} h \pm \mathrm{d} u=0 .
$$

The initial conditions are zero velocity and unit dimensionless height and length at $t=0$. Also, the velocity at $x=0$ is zero, and an additional condition is needed at the nose $x=x_{N}(t)$. As in previous investigations (Ungarish \& Huppert 2002, 2004; Ungarish 2005a), we write

$$
u_{N}=\operatorname{Fr}_{N}^{1 / 2}\left[1-S\left(1-\frac{1}{2} \frac{h_{N}}{H}\right)\right]^{1 / 2} .
$$

Here the Froude 'number' $\mathrm{Fr}$ expresses the ratio of the velocity of propagation of the nose, $u_{N}$, to the effective pressure head (per unit mass) at the nose whose thickness is $h_{N}$. The term in the square brackets of (2.20) is equal to 1 in the non-stratified case $(S=0)$, and smaller than 1 for $S>0$. This term indicates the explicit slow-down of the head due to stratification effects. We argue that the effective $F r$ is a function of $h_{N} / H$ only, and the practical numerical value is approximated well by the simple semi-empirical correlation of Huppert \& Simpson (1980):

$$
F r= \begin{cases}1.19 & \left(0 \leqslant h_{N} / H \leqslant 0.075\right) \\ 0.5\left(h_{N} / H\right)^{-1 / 3} & \left(0.075 \leqslant h_{N} / H \leqslant 1\right) .\end{cases}
$$

We adopt (2.21) as a prototype correlation in the following, but it will be evident that the essence of the analysis and conclusions are not affected by the details of the functional form of $\mathrm{Fr}\left(h_{N} / H\right)$.

The importance of the viscous friction to the motion of the current increases with time and distance of propagation. Even for quite large values of $R e$, the inviscid $\mathrm{SW}$ formulation may become invalid at moderate values of $x_{N}$. This tendency is enhanced by stratification. To monitor this effect, we use the previous results for $u$ and $h$ to estimate the time-dependent ratio of global inertial, $F_{I}$, to viscous, $F_{V}$, effects. Since the inertia per unit volume is represented well by $\rho_{c} u u_{x}$, and the viscous force per unit area is expected to be proportional to $\rho_{o} v u / h$ we obtain, in dimensionless form,

$$
\frac{F_{I}}{F_{V}} \approx \operatorname{Re} \frac{h_{0}}{x_{0}} \frac{\int_{0}^{x_{N}(t)} u u_{x} h \mathrm{~d} x}{\int_{0}^{x_{N}(t)}(u / h) \mathrm{d} x}=\operatorname{Re} \frac{h_{0}}{x_{0}} \theta(t) .
$$

The function $\theta(t)$ is expected to be of the order of unity at the beginning of the propagation and decay to quite small values. This function can be easily calculated from the SW results for $u(x, t)$ and $h(x, t)$, but can also be estimated analytically using box-model considerations.

Assuming that the current is a box of height $h_{N}(t)$, and accordingly $u_{x}=u_{N} / x_{N}$, we obtain $\theta \approx u_{N} h_{N}^{2} x_{N}^{-1}$. The value of $u_{N}$ is estimated from (2.20)-(2.21) as $(a)$ $\left[(1-S) h_{N}\right]^{1 / 2}$ for $S$ not close to 1 ; and $(b) h_{N} / H^{1 / 2}$ for $S \approx 1$. Finally, we use the volume conservation $h_{N}=1 / x_{N}(t)$. This yields

$$
\theta \approx \begin{cases}(1-S)^{1 / 2} x_{N}^{-7 / 2} & (S \text { not close to } 1) \\ H^{-1 / 2} x_{N}^{-4} & (S \approx 1) .\end{cases}
$$


Using these results we can estimate the importance of the viscous terms, and also the limit of validity of the inviscid assumption, for a real gravity current with given $R e$ and $h_{0} / x_{0}$. The inviscid theory is expected to be relevant for, roughly, $F_{I} / F_{V}>3$. The transition from inertial to viscous dominance is not a clear-cut experimental observation because is occurs over a distance of several dimensionless units. The cases discussed in this study are in the inertia-dominated regime.

\subsection{Navier-Stokes considerations}

We consider, again, a two-dimensional rectangular domain, with gravity acting in the negative $z$-direction. The velocity is $\boldsymbol{v}=u \hat{\boldsymbol{x}}+w \hat{\boldsymbol{z}}$. We employ the following dimensional balance equations:

1. conservation of volume

$$
\nabla \cdot v=0
$$

2. momentum balance

$$
\rho \frac{\mathrm{D} \boldsymbol{v}}{\mathrm{D} t}=-\nabla p-\left(\rho-\rho_{b}\right) g \hat{z}+\mu \nabla^{2} \boldsymbol{v},
$$

where $p$ is the pressure reduced with $\rho_{b} g z$ and $\mu$ is the dynamic viscosity coefficient, assumed constant and equal for both fluids;

3. density transport

$$
\frac{\mathrm{D} \rho}{\mathrm{D} t}=0 .
$$

In the numerical computations a small diffusion term, $\kappa \nabla^{2} \rho$, was added to the righthand side of (2.26). This was done for smoothing purposes, but its effect on the results is within the bounds of the numerical truncation errors.

For energy considerations, we multiply the horizontal and vertical components of (2.25) by $u$ and $w$, respectively and add the results. After some algebra and use of (2.24), we obtain

$$
\rho \frac{\mathrm{D}}{\mathrm{D} t}\left[\frac{1}{2}\left(u^{2}+w^{2}\right)\right]=-\nabla \cdot p \boldsymbol{v}-\left(\rho-\rho_{b}\right) g w+\Phi,
$$

where $\Phi$ is the dissipation function $\left(=2 \mu e_{i j} e_{i j}\right.$, where $\left.e_{i j}=(1 / 2)\left(\partial u_{i} / \partial x_{j}+\partial u_{j} / \partial x_{i}\right)\right)$.

Consider the integral of (2.27) over the volume $\Omega$ of the two-fluid system. For simplicity, we consider a closed rectangular domain $0 \leqslant x \leqslant x_{\mathrm{w}}, 0 \leqslant z \leqslant H$. This domain $\Omega$ is the union of $\Omega_{c}$ (for the current) and $\Omega_{a}$ (for the ambient fluid). Within the Boussinesq approximation $O(\epsilon)$ error bounds, the slightly varying $\rho$ on the lefthand side can be replaced by the constant $\rho_{o}$. The integrated left-hand side term thus yields the rate of change of the total kinetic energy, defined straightforwardly by

$$
K_{i}(t)=\rho_{o} \int_{\Omega_{i}} \frac{1}{2}\left(u^{2}+w^{2}\right) \mathrm{d} V,
$$

where $i$ is $a$ for the ambient, $c$ for the current, and there is no subscript for the whole system. On the right-hand side, the integrated contribution of the pressure term vanishes on account of the boundary conditions. The effect of the dissipation term on the time-dependent energy behaviour of the system enters via

$$
\mathscr{D}(t)=\int_{0}^{t} \mathrm{~d} t \int_{\Omega} \Phi \mathrm{d} V .
$$

The integral of the second term on the right-hand side of (2.27), which represents the work of the buoyancy force, can be manipulated into an informative form as 
follows. To this end, we introduce the vertical upward displacement $\eta(x, z, t)$ of a particle of density $\rho$ in the ambient fluid, from its initial position in the linear density profile. The conservation of density of the particle, combined with (2.3), yields

$$
\eta(x, z, t)=z-\left[\rho_{b}-\rho(x, z, t)\right] \frac{H}{\Delta \rho} .
$$

Consequently,

$$
\begin{aligned}
\int_{\Omega} g\left(\rho-\rho_{b}\right) w \mathrm{~d} V= & g \int_{\Omega_{c}}\left(\rho_{c}-\rho_{b}+\frac{\Delta \rho}{H} z-\frac{\Delta \rho}{H} z\right) w \mathrm{~d} V \\
& +g \int_{\Omega_{a}}\left[\rho_{b}-\frac{\Delta \rho}{H}(z-\eta)-\rho_{b}\right] w \mathrm{~d} V \\
= & \frac{\mathrm{D}}{\mathrm{D} t}\left[g \int_{\Omega_{c}}\left[\left(\rho_{c}-\rho_{b}\right) z+\frac{1}{2} \frac{\Delta \rho}{H} z^{2}\right] \mathrm{d} V\right] \\
& +\frac{D}{D t}\left[\rho_{o} \mathscr{N}^{2} \int_{\Omega_{a}} \frac{1}{2} \eta^{2} \mathrm{~d} V\right]-g \frac{\Delta \rho}{H} \int_{\Omega} z w \mathrm{~d} V,
\end{aligned}
$$

where (2.5) was also used. The last term on the right-hand side vanishes because of the velocity boundary conditions. The first and second terms represent the rate of change of the potential energy of the current and ambient, respectively.

Hereafter, dimensionless variables are used unless stated otherwise. The scaling is provided by (2.14); in particular, the energy is scaled with $(1 / 2) \rho_{o} x_{0} h_{0}^{2} g^{\prime}$ and $\eta$ with $h_{0}$.

As expected, the energy terms for the current are similar to these derived for the SW case. In the SW approximation the contribution of $w$ to the kinetic energy has been discarded. According to (2.31), the second term on the right-hand side, the scaled form of the potential energy of the ambient is

$$
P_{a}(t)=\frac{S}{H} \int_{\Omega_{a}} \eta^{2} \mathrm{~d} V
$$

This indicates that for weak stratification (small $S$ ) most of the energy transferred to the ambient is of kinetic type, and hence more prone to viscous dissipation. This trend is consistent with the observation of Necker et al. (2005) that higher levels of kinetic energy are associated with larger values of viscous dissipation.

We are concerned with the behaviour of the mechanical energies; we refer to the sum of kinetic and potential components as total energy. The total energy of the two-fluid system is expected to decay due to irreversible viscous dissipation.

The numerical solution of the NS formulation was obtained by using a finitedifference code. The details are as reported in Ungarish \& Huppert (2004) and here extensions for the calculation of the energy are incorporated.

For the convenience of both numerical simulation and presentation of the results, we introduce the density function $\phi(\boldsymbol{r}, t)$ defined by

$$
\phi(x, z, t)=\frac{\rho(x, z, t)-\rho_{o}}{\rho_{c}-\rho_{o}}=\frac{1}{\epsilon}\left[\frac{\rho(r, z, t)}{\rho_{o}}-1\right],
$$

where $\epsilon$ is the reduced density difference given by (2.1). We expect $0 \leqslant \phi \leqslant 1$, with $\phi=1$ in the domain of the 'pure' dense fluid and $0 \leqslant \phi \leqslant S$ in the domain of the ambient fluid. 
The parameters of the numerical computation are: first, as in the SW formulation, the values of $S$ and the (dimensionless) depth $H$; and, in addition, the values of $R e=\left(g^{\prime} h_{0}\right)^{1 / 2} h_{0} \rho_{o} / \mu, \epsilon$, the initial aspect ratio of the dense fluid $h_{0} / x_{0}$, and length of the tank, $x_{\mathrm{w}}$ (scaled with $x_{0}$ ). (The coefficient of the artificial diffusion term which was added on the right-hand side of the density transport was typically $(10 R e)^{-1}$. The Schmidt number, $\nu / \kappa$, is significantly larger in a typical two-liquid system, but the physical diffusion process is beyond the resolution of the present computations.)

The initial conditions at $t=0$ are

$$
\boldsymbol{v}=\mathbf{0} \quad\left(0 \leqslant x \leqslant x_{\mathrm{w}}, \quad 0 \leqslant z \leqslant H\right)
$$

and

$$
\phi= \begin{cases}1 & (0 \leqslant x \leqslant 1, \quad 0 \leqslant z \leqslant 1) \\ S(1-z / H) & \text { (elsewhere). }\end{cases}
$$

The finite-difference results provide the values of $u, w$ and $\phi$ at grid points. This allows the calculation of the potential and kinetic energies of the current and of the ambient. The values of $\eta_{i, j}$ (at grid points $x_{i}, z_{j}$ ) were calculated with the aid of (2.30) and (2.33) by

$$
\eta_{i, j}=z_{j}+H\left(\phi_{i, j} / S-1\right) .
$$

\section{Results}

We consider the influence of the stratification, $S$, on the energy behaviour of the gravity current released from behind a lock in a configuration with $H=3$ (dimensionless). This geometry is expected to be typical of currents in a non-shallow ambient, and is also compatible with experimental runs 5 and 19 of M. The results are presented in dimensionless form subject to the scaling (2.14).

The SW governing equations (2.16)-(2.17) with the appropriate boundary conditions were solved by a two-step Lax-Wendroff method (see Morton \& Mayers 1994; Press et al. 1992). The typical grid has 200 intervals over $\left[0, x_{N}(t)\right]$ and a time step of 0.04 . The resulting discrete values of $h$ and $u$ were used to calculate the energy integrals (2.11) and (2.12) by the trapezoidal rule. The estimated numerical errors are less than $1 \%$. The predicted propagation of the nose is presented in figure 2 . As expected, as the stratification parameter $S$ increases, the speed of propagation is reduced. Cases $S=0$ and $S=0.29$ are supercritical with respect to $u_{\mathrm{w}}$, and cases $S=0.72$ and $S=1$ are subcritical, see (2.6). Note that the configurations for $S=0.29$ and $S=0.72$ discussed here correspond to experimental runs 5 and 19 respectively of M. Their super- and subcriticality has been confirmed experimentally.

Figure 3 shows the expected decay of the ratio of inertial to viscous effects. The present inviscid approach is valid as long as $\theta \operatorname{Re}\left(h_{0} / x_{0}\right)$ remains large (say $\left.>3\right)$. Thus, for the typical value of $\operatorname{Re}\left(h_{0} / x_{0}\right)=10^{3}$, viscous effects may become dominant after a propagation to about $x_{N}=7$ for small $S$ and $x_{N}=5$ for $S$ close to 1 . We shall restrict our analysis to these values. The constant slope of $\theta$ as a function of $x_{N}$ on the $\log -\log$ plot confirms the estimates (2.23). Figures 4 and 5 display the SW energy balances of the current as functions of time and of distance for various values of the stratification parameter $S$. The energies of each system are referred to the initial potential energy $P_{c}(0)$, see (2.13). In all cases, the potential energy decays monotonically, while the kinetic energy has an initially increasing and then decreasing profile with a maximum of about 0.3 at $t \approx 2$ to 3 . A similar behaviour has been observed by Necker et al. (2005) in the context of homogeneous and particle-driven gravity currents. The 


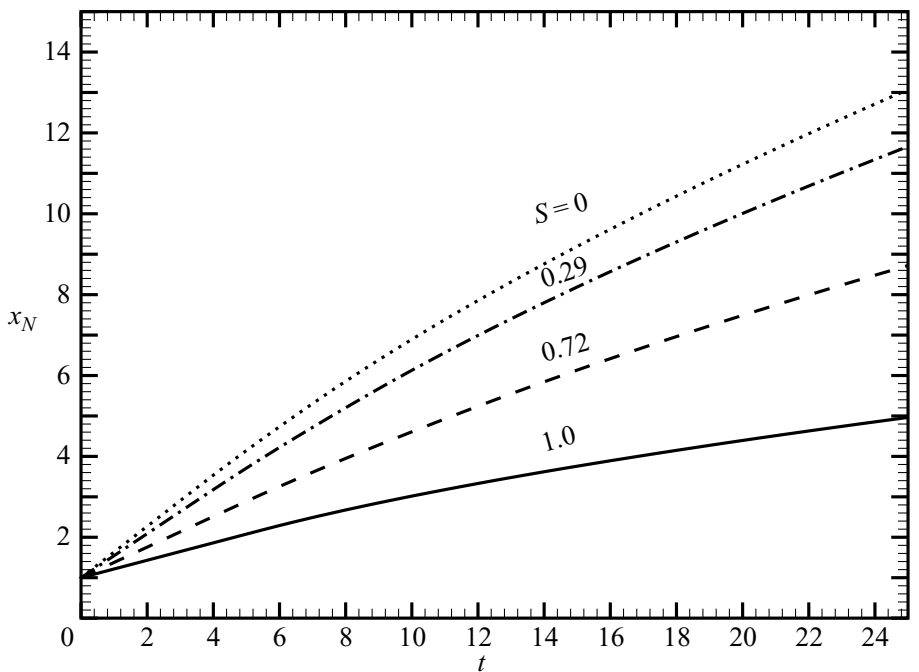

FIGURE 2. SW results. The propagation of the nose as a function of time for $H=3$ and various values of $S$.
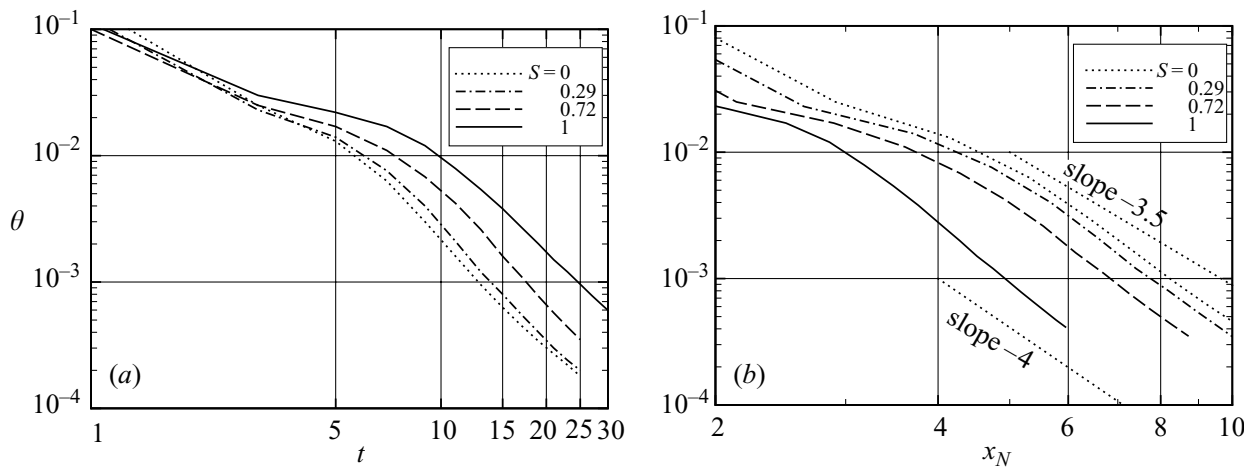

FiguRE 3. SW results. The coefficient $\theta$, see (2.22), for $H=3$ and various values of $S:(a)$ as a function of time; and $(b)$ as a function of $x_{N}$, displaying also the slopes of approximation (2.23).
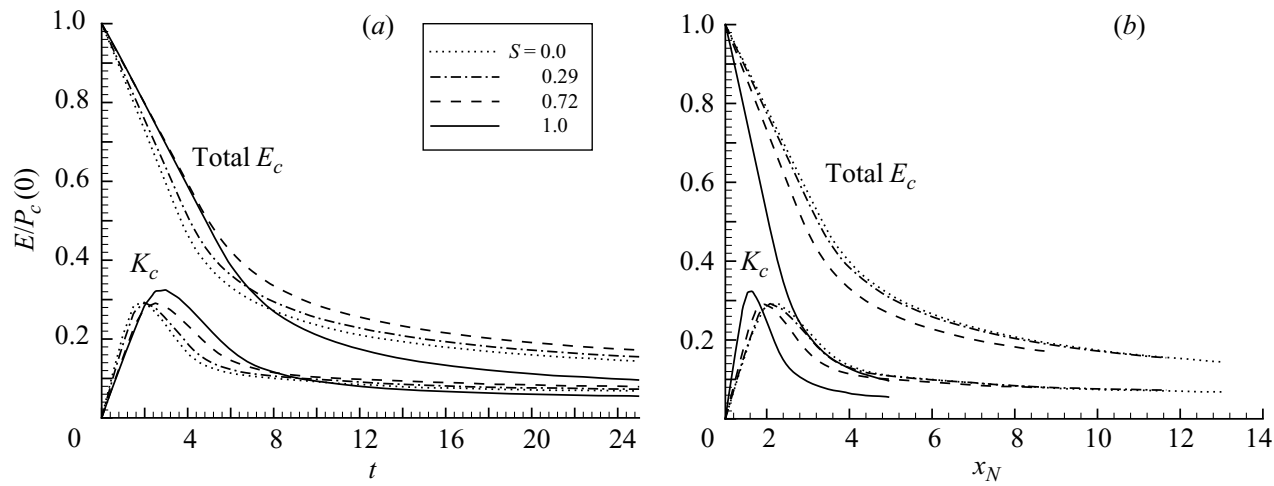

FIGURE 4. SW results. The kinetic and total energy of the current as functions of $(a)$ time and $(b) x_{N}$, for various values of $S$. 

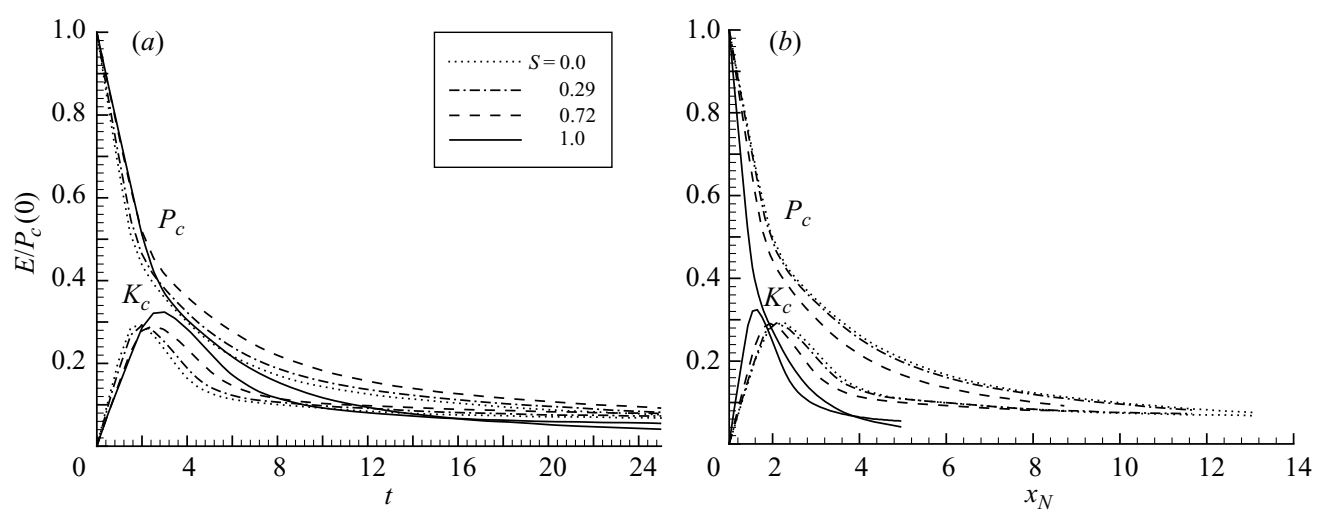

Figure 5. SW results. The kinetic and potential energy of the current as functions of (a) time and $x_{N}(b)$, for various values of $S$.

kinetic energy of the current develops from zero during the 'dam-break' phase of the motion (Ungarish 2005a). The expansion wave which travels backward into the bulk of stationary fluid sets this fluid into motion and increases the kinetic energy. Then the current evolves to a rectangle of height $h_{N}$, approximately; here the kinetic energy reaches the maximum. This happens before the end of the slumping phase of constant-velocity propagation (Huppert \& Simpson 1980; Ungarish \& Zemach 2005). The ratio of kinetic to potential energy becomes close to 1 at a quite advanced stage of propagation $(t \approx 20)$, when the potential energy has decayed to about $10 \%$ of its initial value.

Interesting and unexpected is the fact that the behaviour of energy decay as a function of the distance of propagation $x_{N}$ is quite insensitive to the value of $S$ for $S<0.7$ (say). The case $S=1$ (i.e. $\rho_{c}=\rho_{b}$, the strongest stratification in the present framework) displays a different behaviour with $x_{N}$ to the other cases: the total energy of the current, $E_{c}=P_{c}+K_{c}$, decays faster, and the maximum of the kinetic energy is attained after a shorter propagation. An interpretation is as follows. The total energy of the current decreases with time because of the work of pushing the nose against the ambient pressure. An estimate gives $\mathrm{d} E_{c} / \mathrm{d} x \approx-u_{N}^{2} h_{N}$. Using (2.20) and (2.13) we find that: $(a)$ for $S$ not close to $\left.1,\left[\mathrm{~d} E_{c} / \mathrm{d} x\right) / P_{c}(0)\right] \approx-h_{N}^{2}$; and $(b)$ for $S \approx 1$, $\left.\left[\mathrm{d} E_{c} / \mathrm{d} x\right) / P_{c}(0)\right] \approx-(3 / 2) h_{N}^{3}$. These approximations explain the collapse of the curves $S=0,0.29,0.72$, as opposed to the faster decay curve $S=1$, in figure 5 . The maximum kinetic energy is attained, as explained above, close to the end of the slumping phase. Since the slumping distance is significantly shorter for the case $S=1$, the maximum of kinetic energy occurs at a smaller $x_{N}$ compared to the other cases.

The corresponding NS computations were performed on grids of $320 \times 200$ intervals. In these simulations $H=3$ and $h_{0} / x_{0}=0.25, x_{\mathrm{w}}=8, R e=9.62 \times 10^{3}$ and the values of $S$ were $0,0.29,0.72$ and 1 . The values of $\epsilon$ were $0.115,0.115,0.0804,0.115$, respectively. These parameters were chosen so that the second and third cases reproduce experimental runs 5 and 19 of $\mathrm{M}$. We verified numerically that variations of several percent in the small parameter $\epsilon$ have an insignificant effect on the results (but this must be taken into account in the scaling $g^{\prime}$, of course). This is as expected for a Boussinesq system.

The propagation of the current is considered for the time (or distance) during which the influence of the wall at $x_{\mathrm{w}}=8 \mathrm{can}$ be neglected and during which the inertia effects are dominant (i.e. $\operatorname{Re}\left(h_{0} / x_{0}\right) \theta>3$ ). In contrast to the SW model, whose numerical 

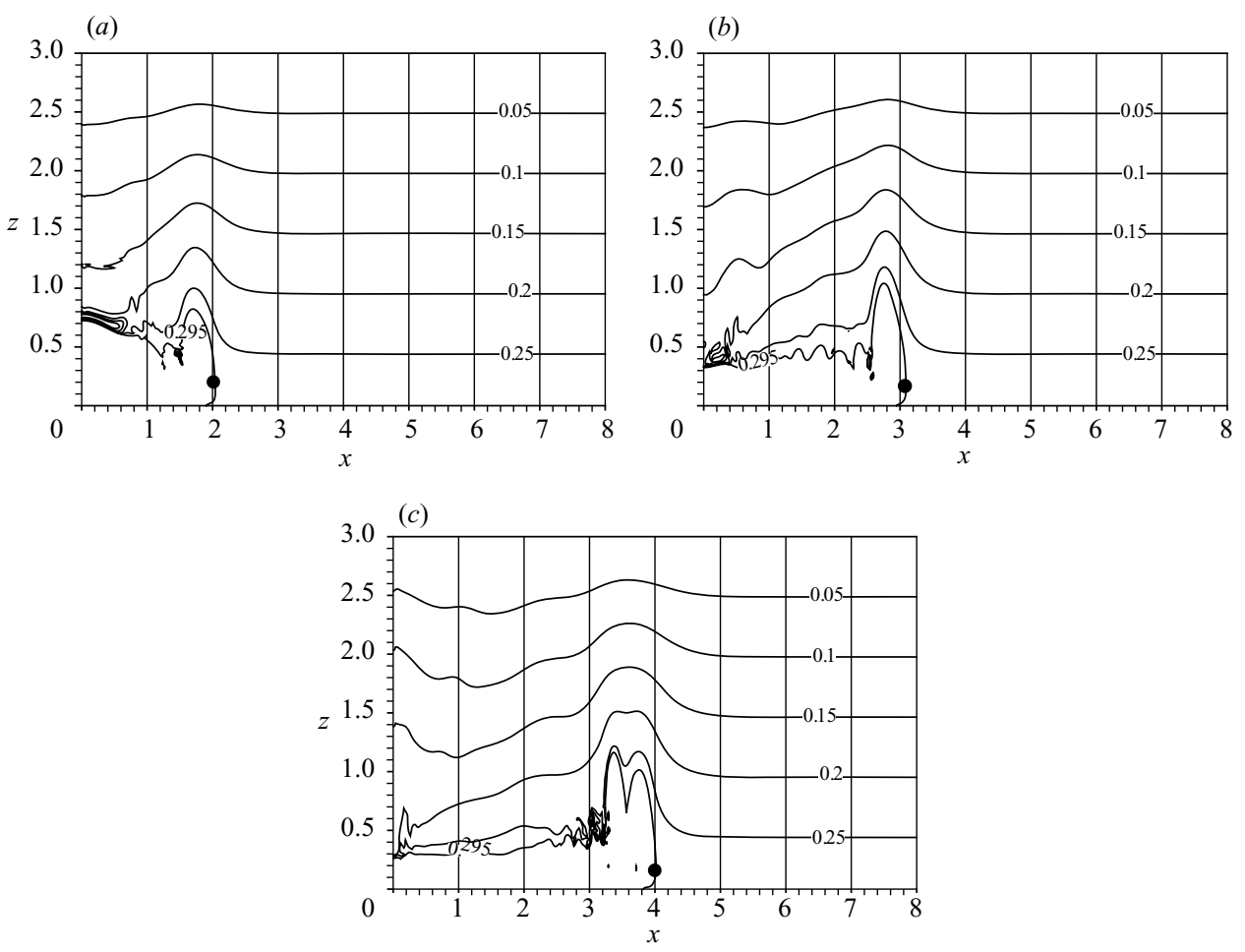

FiguRE 6. NS results. Density contour lines at various times for $S=0.29$. The solid circle marks the 'nose' of the current, defined as the foremost forward point of the dense fluid. (a) $t=2$, (b) $t=4$, (c) $t=6$.

solution required insignificant computer resources and the analysis of the data was straightforward, the NS simulation required significant CPU-time and storage space, and a serious effort to process the data.

Typical flow-field results of the NS simulations are shown in figures 6 and 7. We see that the shape of the current is very complex in the region of the nose, but the tail domain is fairly simple. The stronger stratification (figure 7) reduces the height of the head compared to the case of weaker stratification (figure 6). The isopycnals of the ambient fluid are displaced considerably above the head of the current. For $S=0.72$ (typical for subcritical currents) a strong wave-head interaction occurs (see also $\mathrm{M}$ and Ungarish \& Huppert 2004). This is further elucidated in figure 8 . We see that at $t \approx 12$ the velocity of propagation enters a state of strong deceleration, which practically brings the nose to rest at $t \approx 14$. However, at $t \approx 16$ the original speed is recovered. This behaviour has been recorded in the experiments of $\mathrm{M}$, and it is reproduced well by our numerical code. The SW theory does not capture this effect. The behaviour displayed by these figures gives rise to searching questions in the context of the present investigation: is the simplified SW theory able to capture the energy balances; what happens to the energy of the current in the special stage of interaction with the ambient; and, moreover, is the SW energy balance valid after this occurrence?

A major object of the present investigation was the verification of the SW energy behaviours against the corresponding NS results. The pertinent comparison is shown in figure 9. We observe that the SW and NS results are fairly close for a significant period of propagation. Eventually, the NS results decay more quickly to zero than 

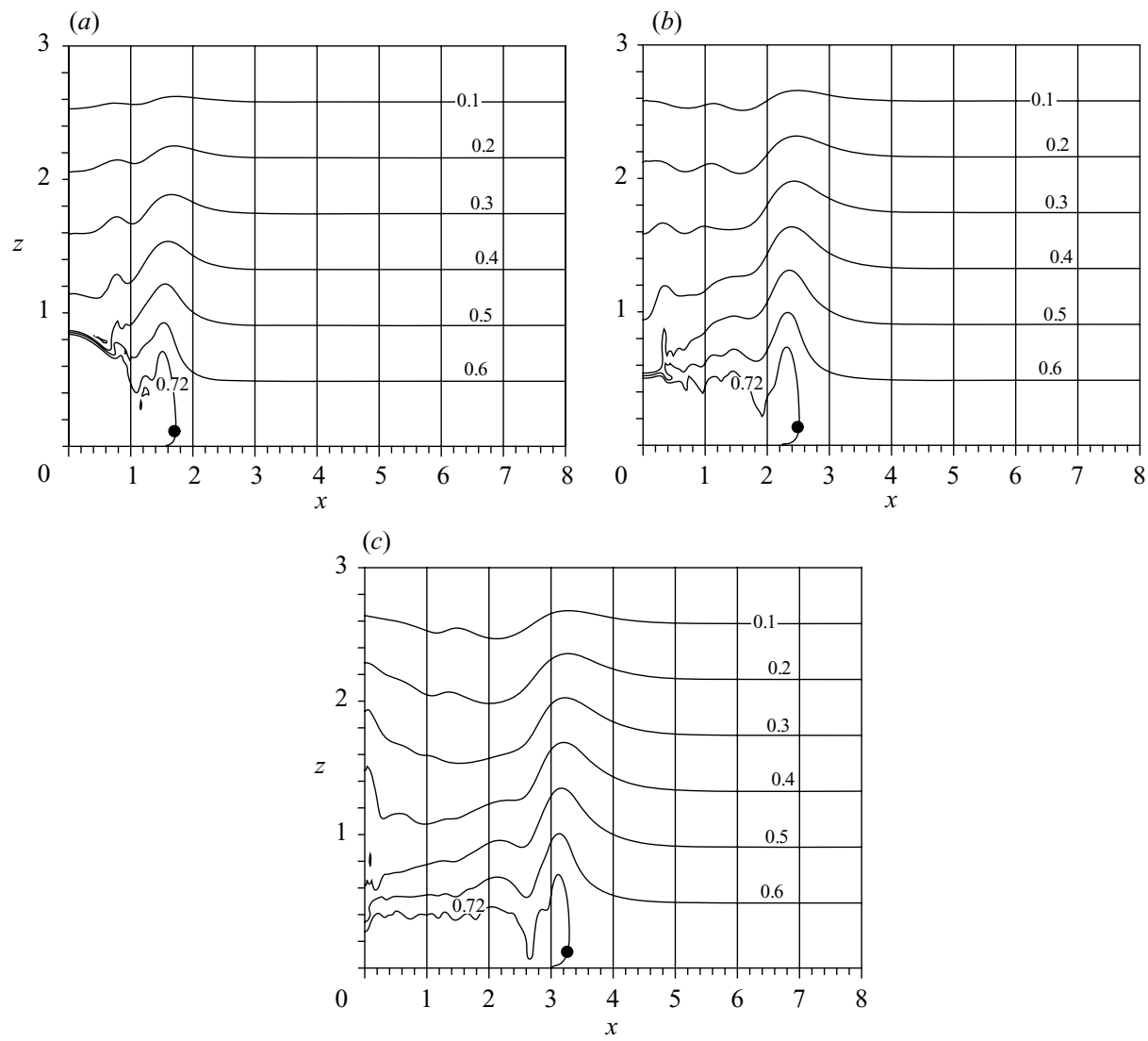

Figure 7. NS results. As figure 6 but for $S=0.72$. (a) $t=2$, (b) $t=4$, (c) $t=6$.

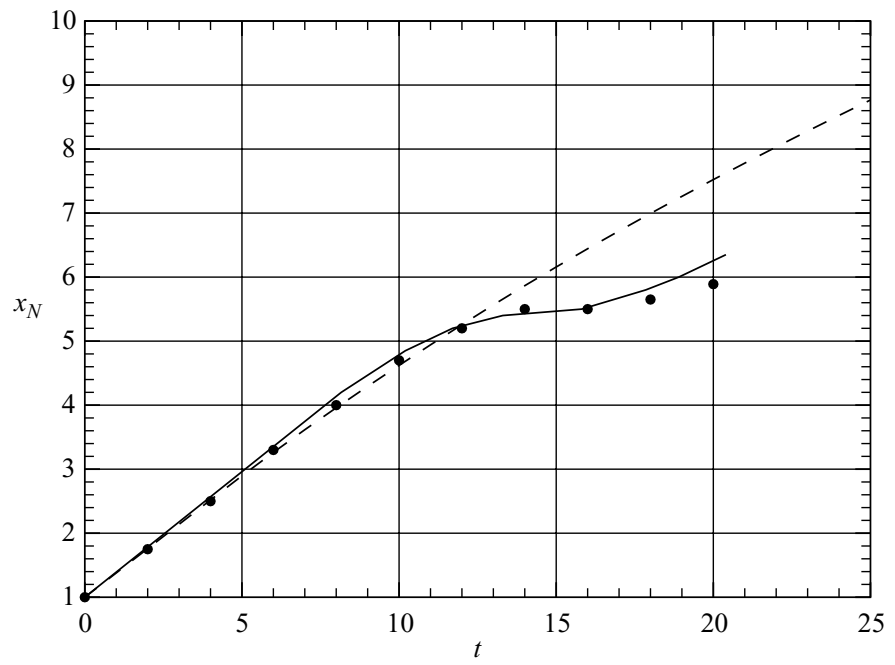

FiguRE 8. $x_{N}$ as a function of time for $S=0.72$. Results from experimental Run 19 of M (full line), SW (dashed line) and NS (symbols). 

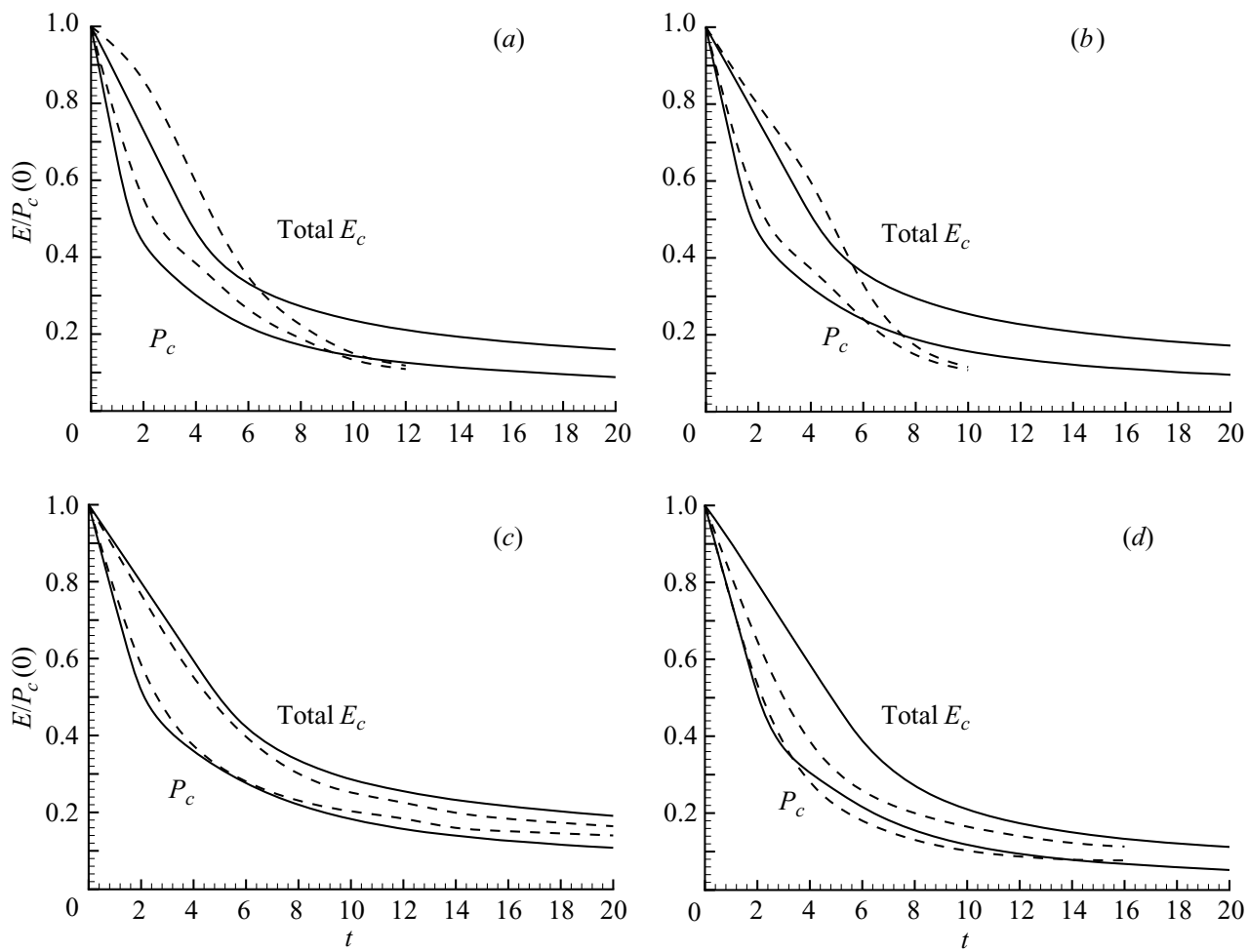

Figure 9. The potential and total (mechanical) energy of the current as functions of time. Comparisons of NS (full lines) and SW (dashed lines) results. (a) $S=0$ (unstratified), (b) 0.29 , (c) 0.72 and $(d) 1.0$.

the SW predictions. This is a result of viscous effects, which are not included in the SW model. For weak stratifications, shortly after release, the potential and total energy of the current as predicted by the NS simulations are slightly larger than those determined by the SW results. This can be attributed to the initial adjustment motion of the head which is incompatible with the SW assumptions. We conclude that, overall, the NS computations confirm the energy predictions of the simplified SW model. This strengthens confidence in the insights derived into the effects of stratification.

Additionally, an interesting outcome is that the wave-nose interaction in the subcritical current has no significant effect on the energy exchange. This is illustrated by the configuration for $S=0.72$. The NS simulations show clearly a change of behaviour of $x_{N}(t)$ in the time interval 10 to 14 , see figure 8 . However, the corresponding graphs of energy as a function of time, produced by the same NS simulations, do not display a change of behaviour during this time interval. An interpretation is as follows. The interaction occurs when the kinetic energy of the current is already low (see figure 5) and the head is relatively shallow. In these circumstances, a relatively small increase of the velocity of the main bulk which follows the head is able to recover the energy lost by the head during the deceleration phase. Afterward, this process is reversed and the head regains its velocity. This is an encouraging result regarding the predictive ability of the SW model. This model does not detect the interaction, and hence it could be argued that its predictions become invalid after this occurrence. However, we found that the energy balances of a real current are also unaffected by this interaction. Thus, the prediction of energy as a 


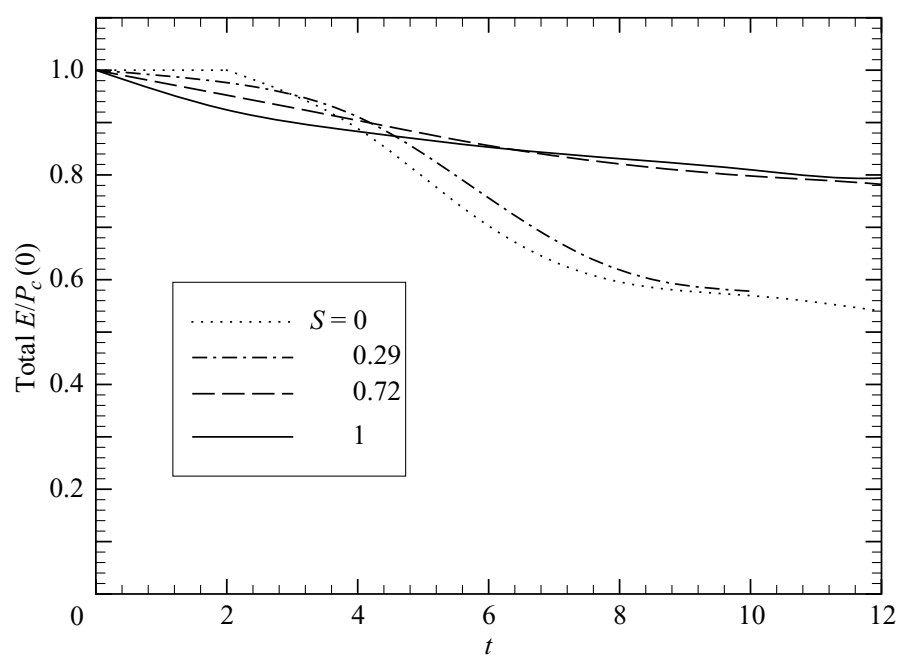

FIGURE 10. Total mechanical energy of the two-fluid system as a function of time for various $S$, NS results.
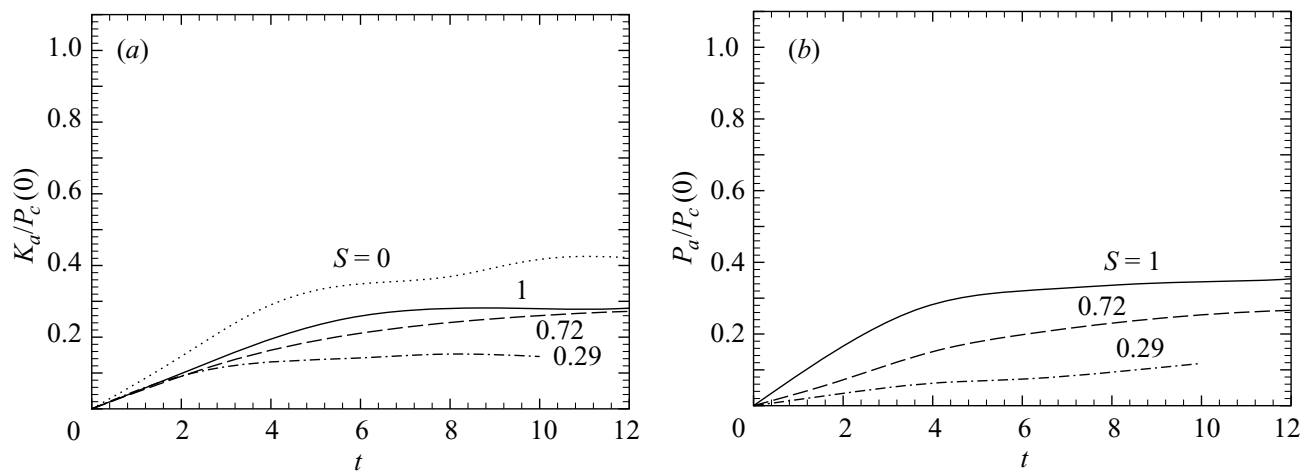

FIgURE 11. (a) Kinetic and $(b)$ potential energy of the ambient as a function of time for various $S$, NS results. (The potential energy result for $S=0$ is zero.)

function of time by this model remains valid for a longer period of time than could be anticipated for a subcritical propagation.

Figures 10 and 11 provide information on the total mechanical energy (kinetic plus potential) of the two-fluid system and of the energies of the ambient, obtained from the NS computations. Evidently, the total (mechanical) energy is not conserved because of irreversible viscous dissipation. We note that stratification hinders the decay of the total mechanical energy. This is consistent with the observation of $\mathrm{M}$ that the interface of the current becomes smoother when stratification increases. An interpretation can be inferred from the behaviour of the kinetic and potential energies in the ambient. The stratification enhances the ability of the ambient to accumulate potential energy. Thus, as $S$ increases, the ratio of kinetic to potential energy in the ambient is reduced. For $S=0.72$ the ratio of potential to kinetic energies in the ambient is typically 1 , while for $S=0.29$ the ratio is typically 0.5 . This reduces the velocity differences in the ambient field and hence also the viscous dissipative friction.

Considering the behaviour of the total mechanical energy of the system as a function of time, we notice a striking similarity between the supercritical currents 
$S=0$ and $S=0.29$, on one hand, and between the subcritical currents $S=0.72$ and $S=1$, on the other hand.

\section{Concluding remarks}

The shallow-water analysis presented here, which neglects motion in the ambient, seems to capture well the energy exchange of the gravity current in the inertiadominated stage of propagation. In particular, this approach is able to elucidate by comparatively simple means the effects of stratification on the energy balances of the gravity current. This indicates that for sufficiently deep currents ( $H \geqslant 3$ say), the motion in the ambient has little influence on the energetic behaviour of the dense fluid. In other words, the ambient reacts to, rather than interacts with, the motion of the dense fluid.

We found that super- and subcritical currents differ in their energetic behaviour. The latter group maintains the total energy for a longer dimensionless time.

The propagation of the subcritical current is subject to interactions of the head with the waves, during which the velocity of the head decreases significantly and then increases. However, the NS simulations (which detect this effect well) indicate that no significant counterpart effect occurs in the energy exchange behaviour. The SW model provides valid energy results as a function of time during this problematic stage, in spite of the fact that it does not incorporate the interaction effect.

The major limitation of the SW energy balances used in our investigation is imposed by the neglected viscous friction. This effect cannot be easily incorporated into the analysis. We have presented a criterion for estimating the limit of validity of the inviscid approach, but the understanding of the energy exchanges thereafter requires a special investigation. This topic is left for future work.

The present results are for a rectangular two-dimensional geometry. We expect that the main insights are valid for more general cases, but the details must be worked out. For example, an extension to axisymmetric configurations is an interesting topic. In this case, a slumping phase with constant velocity of propagation does not develop, and this may affect the behaviour of the kinetic energy of the current. Moreover, divergent geometry is expected to bring viscous forces into play at an earlier stage of propagation. This topic is also left for future work.

The research was supported by EPSRC. The research of H. E. H. is supported by a Royal Society Wolfson Merit Award.

\section{REFERENCES}

Amen, R. \& Maxworthy, T. 1980 The gravitational collapse of a mixed region into a linearly stratified fluid. J. Fluid Mech. 96, 65-80.

BaInes, P. G. 1995 Topographic Effects in Stratified Flows. Cambridge University Press.

Benjamin, T. B. 1968 Gravity currents and related phenomena. J. Fluid Mech. 31, 209-248.

Faust, K. M. \& Plate, E. J. 1984 Experimental investigation of intrusive gravity currents entering stably stratified fluids. J. Hydraulic Res. 22, 315-325.

FlynN, M. R. \& Sutherland, B. R. 2004 Intrusive gravity currents and internal gravity wave generation in stratified fluid. J. Fluid Mech. 514, 355-383.

Huppert, H. E. 2000 Geological fluid mechanics. In Perspectives in Fluid Dynamics: A Collective Introduction to Current Research (ed. G. K. Batchelor, H. K. Moffatt \& M. G. Worster), pp. 447-506. Cambridge University Press.

Huppert, H. E. \& Simpson, J. E. 1980 The slumping of gravity currents. J. Fluid Mech. 99, 785-799.

KaO, T. W. 1976 Principal stage of wake collapse in a stratified fluid: Two-dimensional theory. Phys. Fluids 19, 1071-1074. 
Manins, P. C. 1976 Mixed region collapse in a stratified fluid. J. Fluid Mech. 77, 177-183.

MaXwORTHY, T. 1980 On the formation of nonlinear internal waves from the gravitational collapse of mixed regions in two and three dimensions. J. Fluid Mech. 96, 47-64.

Maxworthy, T. 1983 Experiments on solitary internal Kelvin waves. J. Fluid Mech. 128, 365-383.

Maxworthy, T., Leilich, J., Simpson, J. E. \& Meiburg, E. H. 2002 The propagation of gravity currents in a linearly stratified fluid. J. Fluid Mech. 453, 371-394 (referred to herein as M).

Morton, K. W. \& Mayers, D. F. 1994 Numerical Solutions of Partial Differential Equations. Cambridge University Press.

Necker, F., Härtel, C., Kleiser, L. \& Meiburg, E. 2005 Mixing and disipation in particle-driven gravity currents. J. Fluid Mech. 545, 339-372.

Press, W. H., Teukolski, S. A., Vetterling, W. T. \& Flannery, B. P. 1992 Numerical Recipes in Fortran. Cambridge University Press.

DE RoolJ, F. 1999 Sedimenting particle-laden flows in confined geometries. PhD thesis, Department of Applied Mathematics and Theoretical Physics, University of Cambridge.

Schooley, A. H. \& Hughes, B. A. 1972 An experimental and theoretical study of internal waves generated by the collapse of a two-dimensional mixed region in a density gradient. J. Fluid Mech. 51, 159-175.

Simpson, J. E. 1997 Gravity Currents in the Environment and the Laboratory. Cambridge University Press.

Ungarish, M. 2005a Dam-break release of a gravity current in a stratified ambient. Eur. J. Mech. B/Fluids 24, 642-658.

UNGARISH, M. $2005 b$ Intrusive gravity currents in a stratified ambient - shallow-water theory and numerical results. J. Fluid Mech. 535, 287-323.

UNGARISH, M. 2006 On gravity currents in a linearly stratified ambient: a generalization of Benjamin's steady-state propagation results. J. Fluid Mech. 548, 49-68.

Ungarish, M. \& Huppert, H. E. 1999 Simple models of Coriolis-influenced axisymmetric particledriven gravity currents. Intl J. Multiphase Flow 25, 715-737.

Ungarish, M. \& HupPerT, H. E. 2002 On gravity currents propagating at the base of a stratified ambient. J. Fluid Mech. 458, 283-301.

Ungarish, M. \& HupPERT, H. E. 2004 On gravity currents propagating at the base of a stratified ambient: effects of geometrical constraints and rotation. J. Fluid Mech. 521, 69-104.

Ungarish, M. \& ZEMACH, T. 2005 On the slumping of high Reynolds number gravity currents in two-dimensional and axisymmetric configurations. Eur. J. Mech. B/Fluids 24, 71-90.

Wu, J. 1969 Mixed region collapse with internal wave generation in a density-stratified medium. J. Fluid Mech. 35, 531-544. 